Buchbesprechung

Professionelle Beziehungen. Theorie und Praxis der Balintgruppenarbeit

Otten $\mathrm{H}$

Heidelberg: Springer Verlag, 2012, 137 S., 39,95€

ISBN 978-3-642-03609-5

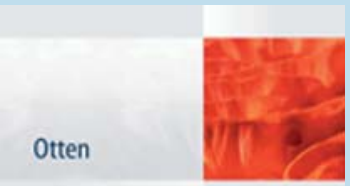

\section{Professionelle Beziehungen}

\begin{tabular}{|c|}
\hline 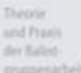 \\
\hline
\end{tabular}

$$
\text { Q Springer }
$$

„Der Arzt, sein Patient und die Krankheit" - dieses medizingeschichtlich bahnbrechende Grundlagenwerk Michael Balints hat den Anfang gemacht: es hat erstmals im Setting der allgemeinmedizinischen Versorgung herausgearbeitet, dass Krankheit eben nicht betrachtet werden kann als Defekt in einer Maschine, sondern dass es nur kranke Menschen gibt, die in ihrer leib-seelischen Ganzheit betroffen sind und entsprechend behandelt werden müssen, bei Strafe der falschen und schädlichen somatischen Medizin im Falle der Missachtung dieses Grundsatzes. Balint hat mit seinem Werk die Beziehung zwischen Arzt und Patient wieder zum Thema gemacht und deutlich werden lassen, welches Potenzial an heilen Kräften, aber auch an Vertiefung des Kranken und Unheilen in dieser Beziehung liegt. Eine wesentliche Folge dieses Werks war die Entstehung der Balintgruppen, heute unentbehrlicher und selbstverständlicher Teil der psychosomatischen Ausbildung und Praxis.

Das vorliegende Werk möchte einen Überblick geben über die Balintgruppenarbeit in ihrer Entstehung und heutigen Praxis. Das Praxiskapitel beschreibt entsprechend den Ablauf der Sitzungen sowie die Balintgruppenarbeit mit somatisch tätigen Ärzten, Psycho-Ärzten (Psychiatern, Psychotherapeuten und
Psychosomatikern) sowie mit Studenten. Die Darstellungen beinhalten des Weiteren aber auch Balintgruppenarbeit und ihre besonderen Voraussetzungen und Schwierigkeiten in unterschiedlichen Berufsgruppen, Kulturen und Nationalitäten. Damit nicht genug, führt die Autorin auch in die Welt der Balintgruppenarbeit mit „zusätzlichen kreativen Elementen“ wie Skulptur, Rollenspiel, Psychodrama oder Imagination ein. Das ist für einen „somatisch tätigen Arzt“ noch weit schwerer zu schlucken als eine Gruppe, in der er seine „apostolische Funktion" abgeben muss, aber doch ein Faszinosum, das zu erfahren wohl lohnend sein könnte.

Der Bildungsweg zu einer Leitung von Balintgruppen ist im Detail gezeichnet, sowohl hinsichtlich der formalen Voraussetzungen als auch der weiteren Ausbildung einschließlich Supervision der Gruppenleiter.

Die Darstellungen sind knapp, zuweilen sicherlich zu knapp, aber immer präzise. Immerhin hätte man sich mehr Beispiele aus der Praxis gewünscht, doch allein dieser Wunsch zeigt schon an, dass es der Autorin gelungen ist, das Interesse des Lesers für die Balintgruppenarbeit zu wecken. Eindeutig zu kurz ausgefallen ist die Zusammenfassung der Forschungsergebnisse, die nur 11 Seiten umfasst, ebenso ist das Literaturverzeichnis sehr karg.

Dessen ungeachtet: Wer sich über die Balintgruppenarbeit informieren möchte, ist als „somatisch tätiger Arzt“ ebenso wie als „Psycho-Arzt“, aber auch als Interessierter aus nichtmedizinischen Berufen mit diesem Band sehr gut bedient.

Prof. Dr. Santiago Ewig, Bochum 